\title{
Les enjeux énergétiques et le développement régional
}

\author{
Jean Matuszewski ${ }^{1}$ \\ E\&B DATA
}

Discours prononcé aux Assises de l'énergie

Saguenay (Québec), le 30 mars 2005

J e vais vous entretenir aujourd'hui de la question énergétique, mais sous un angle particulier, celui du choix de site d'investissement des entreprises pour lesquelles les coûts d'électricité constituent une part élevée des coûts de production. Je vais tenter d'indiquer les possibilités et les limites de ce que les régions peuvent attendre de ces entreprises - puis au-delà, comment les régions peuvent espérer aller encore plus loin dans leur développement.

\section{Crise de l'énergie}

Le débat de ce matin, l'envolée actuelle des prix des produits pétroliers et la certitude croissante que ceuxci vont demeurer élevés, l'essor des initiatives concernant les énergies alternatives, tout l'indique, nous vivons, au Québec et ailleurs dans le monde, ce qui est en train de devenir une crise de l'énergie. Du côté de l'offre : les sources d'énergie traditionnelles s'é-
Nous vivons, au Québec et ailleurs dans le monde, ce qui est en train de devenir une crise de l'énergie.
Québec ne seront jamais plus concurrentiels par rapport aux prix d'autres sources d'énergie. Je note en plus que l'hydroélectricité est une énergie renouvelable et qu'enfin, le mode de propriété est public. C'est-à-dire que, comme contribuables, nous avons payé le développement des infrastructures et qu'en retour, nous bénéficions d'une tarification avantageuse et d'une souplesse dans l'application de celleci. Tel a du moins été le pacte qui nous a servis depuis la nationalisation de l'électricité lors de la Révolution tranquille, et par nous j'entends : citoyens, entreprises et régions. Qu'en est-il du développement économique du Québec et de ses régions dans cette nouvelle donne ? Que recherchent les entreprises ? Quels sont leurs facteurs de localisation?

En supposant que l'énergie redevienne disponible, et cela va se faire d'ici trois ou quatre ans, peut-on attirer encore les entreprises énergivores au Québec ? Peut-on les conserver? Que recherchent les entreprises ? Parlons donc des facteurs de localisation dans le cas particulier des projets énergivores. Pour fins de discussion, je qualifie puisent. Du côté de la demande : l'émergence de nouveaux pays industrialisés, l'enrichissement rapide en Asie et en Europe de l'Est multiplient le nombre de consommateurs d'énergie, qu'il s'agisse d'individus ou d'entreprises. Résultat : une crise généralisée qui affecte les consommateurs, les gouvernements, les entreprises. La crise, cependant, se vit différemment selon les pays et les régions. Chez nous, vous le savez, la mauvaise nouvelle, c'est que nos réserves énergétiques sont sous forte pression. La bonne, c'est que la situation est conjoncturelle et que la situation devrait graduellement commencer à s’améliorer à partir de 2008. Les potentiels énergétiques les plus avantageux ont sans doute déjà été exploités, mais il existe encore un potentiel à exploiter et, à cet égard, je crois qu'il est prématuré de décréter que les coûts marginaux d'électricité au d'énergivores les productions industrielles dont les coûts énergétiques représentent $20 \%$ et plus des frais variables.

- Une première catégorie de facteurs de localisation concerne les aspects physico-techniques, soit ceux qui qualifient un site donné selon les exigences de l'industrie et du projet en question en termes d'infrastructures de transport, de disponibilité d'énergie et de sécurité de l'approvisionnement, d'exigences immobilières spéciales, de capacité d'accéder à une main-d'œuvre spécialisée, etc. Les sites qui ne se qualifient pas à cet égard sont en général éliminés d’office par les investisseurs, même si ces sites avaient pu par ailleurs fournir quelques avantages de coûts. 
- Les sites qui se qualifient sur le plan physicotechnique sont ensuite examinés à partir d'une deuxième série de facteurs de localisation, celui des coûts. Coûts de main-d'œuvre pour l'exploitation mais aussi la construction, coûts de transport et d'accès aux marchés, fiscalités diverses (provinciale, locale, taxes sur le capital), impact des réglementations et, bien sûr, le coût de l'énergie. Plus ce dernier est élevé dans les frais variables de l'unité de production envisagée, plus il pèsera lourd dans la décision. Mais il ne faudrait pas faire l'erreur de croire que le coût de l'énergie est l'unique déterminant de la décision. Le calcul financier, qui se fait en effet sur l'ensemble des coûts, incluant la construction, la fiscalité et intègre la prévision sur un horizon de 5, 10 ou 20 ans, aboutit sur un calcul du retour sur l'investissement qui est comparé à celui résultant d'analyses comparables pour des sites alternatifs. En bout de ligne, les différents avantages ou désavantages de coûts sont donc intégrés pour permettre un calcul global. Autrement dit, on calcule dans les états financiers pro forma le retour sur l'investissement dans les différents sites et on choisit en principe le site le plus rentable à partir d'anticipations sur l'évolution des revenus et des coûts sur la période de calcul.

Une enquête réalisée en 2004 auprès d'entreprises industrielles énergivores l'indique, l'énergie (sa disponibilité et ses coûts) devient un facteur de localisation encore plus important qu'il n'était par le passé. Tant sur le plan physico-technique que sur le plan des coûts, l'énergie continue à jouer un rôle clé et plus important que la proximité des marchés, comme l'indique par exemple le choix récent de nouvelles alumineries en Afrique du Sud, dans le Golfe persique et en Islande. Mais un profil avantageux sur le plan de l'énergie n'assure pas le choix final d'un site à n'importe quelle condition.

- En effet, le choix final ne se fait pas sur la base des deux catégories de facteurs énoncées plus haut (les facteurs physico-techniques et les facteurs de coûts). Le choix final peut en effet être influencé, voire renversé par des facteurs intangibles. Alors que l'ensemble du processus est un exercice rigoureux sur le plan technique et comptable, autant la décision finale repose sur des intangibles. J'illustre par un cas extrême : le dirigeant à qui l'on propose un projet nettement avan- tageux sur le plan des coûts décidera peut-être de ne pas aller de l'avant s'il a des raisons de croire qu'une nationalisation de ses installations est possible. Ou encore s'il a des raisons de s'attendre à des lignes de piquetage sur son chantier de construction. Ou pour prendre un cas moins extrême, le dirigeant pourra décider de ne pas aller de l'avant s'il anticipe que les « règles du jeu » (ex. : coûts, fiscalité) peuvent changer, de façon importante et subite. En effet, les investisseurs ne cherchent pas tant le meilleur climat d'investissement que celui qui s'améliore graduellement, et surtout, qui est prévisible. La stabilité de l'environnement réglementaire ou fiscal, les relations de travail, l'accueil de la communauté sont donc des éléments critiques. Il est difficile de surestimer l'importance de la stabilité et de la prévisibilité pour des investisseurs qui immobilisent des milliards de dollars et qui ont des comptes à rendre à des actionnaires qui, eux s'attendent à une performance élevée à court, à moyen et à long termes. Est-il nécessaire de conclure que les sites qui ne présentent pas d'assurances raisonnables quant à la stabilité ou la prévisibilité des coûts énergétiques ne mettent pas les chances de leur côté ? En bout de ligne, entre les sites acceptables sur le plan physico-technique, entre les sites qui sont équivalents sur le plan des coûts, ce seront les éléments intangibles qui feront la différence.

Pour résumer, la disponibilité d'énergie à des coûts compétitifs est donc un facteur de localisation critique, mais pas à n'importe quelle condition. Voilà donc pour les attentes initiales de l'investisseur. Qu'en est-il des attentes des communautés d'accueil ? Ces investissements sont-ils désirables?

\section{L'énergie (sa disponibilité et ses coûts) devient un facteur de localisation encore plus important qu'il n'était par le passé.}

\section{Les attentes des communautés envers les entreprises}

Examinons d'abord la question de la désirabilité de ces projets. Qu'est ce qu'on recherche avec la venue et le maintien de ces investissements ? Quelles sont les attentes des communautés ? Celles-ci recherchent : 
Sur le plan économique :

- les salaires (et avantages sociaux) et le pouvoir d'achat que ceux-ci diffusent dans la communauté;

- les taxes locales qui permettent la fourniture de services publics à la population ainsi que le développement et le maintien d'infrastructures.

Sur le plan du développement durable, les communautés recherchent le respect des règles et des principes environnementaux, en commençant par la sécurité des travailleurs et de la population.

Ce sont donc des critères de base. Parfois, on peut aller plus loin et l'investisseur peut fournir les contributions additionnelles suivantes :

- la contribution à structurer un tissu industriel local (amont, aval);

- la contribution à un système d'innovation régional (incluant la recherche mais aussi la formation et les transferts avec les universités, les collèges et les PME);

- la contribution à la cohésion sociale, passant par des voies aussi diverses que le soutien aux activités communautaires, ou encore le soutien à l'insertion de groupes défavorisés).

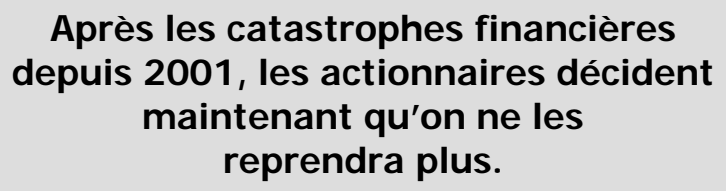

Les entreprises ne vont pas toutes aussi loin dans le soutien à leur communauté d'accueil. Et puis, même si tous ces types de contribution sont réunis, la concurrence ou l'évolution des marchés peuvent changer la donne. Et de ce côté, il semble que les cartes soient contre les communautés d'accueil. Je mentionnerais ici la déception qu'entraînent les réinvestissements dans les modernisations qui amènent souvent des coupures d'emploi. Et comment ne pas mentionner l'alternative dramatique que constitue un niveau inchangé d'emploi doublé d'une absence de réinvestissement, situation qui amène à terme la fin pure et simple de l'activité sur le site en question?

\section{Les attentes des actionnaires envers les entreprises}

Il peut être tentant, lorsqu'on fait partie d'une communauté qui accueille ces entreprises, de se révolter contre cet état de choses. "Les grandes entreprises peuvent faire plus », dira-t-on, "elles doivent faire plus en termes de retombées locales ».

Elles peuvent faire plus ? La réponse n’est pas nécessairement positive. Ce n'est pas parce qu'une usine est installée avec des immobilisations massives et apparemment invulnérables qu'elle n'est pas à risque pour autant. La réalité du monde financier d'aujourd'hui est que les exigences de rentabilité sont devenues de plus en plus élevées au cours des dernières années. Après les catastrophes financières depuis 2001, où les actionnaires se sont laissés piéger par les déclarations ou les rêves de certains dirigeants d'entreprises, les actionnaires décident maintenant qu'on ne les reprendra plus. Deux exemples illustrent le désir des actionnaires de contrôler, de cadenasser même, la valeur ou le rendement des entreprises.

- Premier exemple, les vulture funds, qui achètent des entreprises sous-évaluées et, à coup de discipline opérationnelle d'une rigueur extrême, augmentent la valorisation des entreprises pour leur revente, quelques années après leur acquisition. On les a entre autres vu intervenir récemment dans le secteur de la sidérurgie aux États-Unis.

- Second exemple : les Fiducies de revenus, qu'on retrouve beaucoup dans le secteur énergétique et dans certains produits de base industriels (commodities). Ces fiducies sont actuellement très populaires à cause de leur performance et de leur rendement soutenu. Dans le cadre de ces nouveaux types de structure d'entreprises, des usines sont cédées aux nouveaux actionnaires avec un objectif unique : la rentabilité à court terme. Pas l'objectif principal : l'objectif unique. Qu'en penser ? Avant de juger trop rapidement, veuillez considérer que plusieurs d'entre nous sans doute en bénéficient par le biais de nos placements ou de nos fonds de pension. Résultat pratique : des rendements effectivement élevés pour les fiducies de revenu. Et un objectif de rendement encore plus difficile à atteindre pour les entreprises qui ne sont pas des fiducies, et qui font en plus des efforts d'intégra- 
tion et de structuration industrielle dans leur communauté d'accueil.

Ces deux cas ne sont que des exemples de la pression de rentabilité accrue exercée par les actionnaires auprès des dirigeants des entreprises, puis auprès des dirigeants d'usines. Ces pressions s'accompagnent souvent de choix déchirants dans l'allocation des ressources humaines et financières. On ne peut demander sans risque à ces entreprises d'être les sauveurs du niveau de vie des populations en région.

\section{Alors : double t-on les prix d'électricité ?}

Si l'on ramène les prix d'électricité au niveau du marché nord-est américain, notre principal facteur de différentiation positif, sur le plan des coûts, aura disparu. Je fais référence au facteur de différentiation " positif » parce que nous avons aussi des facteurs de différentiation négatifs. Je n’en mentionnerai qu'un ici, et c'est l'éloignement. Pour les régions, il ne s'agit pas seulement d'une question de kilométrage. En effet, nous sommes situés à l'extrême nord du territoire habité en Amérique du Nord, avec tous les désavantages économiques que cela comporte et qui se manifestent par exemple dans les industries des services, qu'il s'agisse de transport ou de services professionnels spécialisés. Nous perdrons donc notre unique facteur de différentiation positif sur le plan des coûts, celui qui nous permet en quelque sorte de compenser nos désavantages dus à l'éloignement, mais surtout à notre localisation aux confins du tissu industriel nordaméricain.

Il est curieux de constater comme
la question de l'impact de la hausse
des tarifs d'électricité sur les régions
n'est pas souvent étudiée au Québec.

À noter par ailleurs qu'il n'y a pas eu depuis au moins une génération de grandes usines «électrivores » construites dans le Nord-Est des États-Unis comparables à celles qui l'ont été au Québec, et la poignée de celles qui subsistent survivent grâce aux tarifs spéciaux qui ont été négociés ou conservés (clauses « grand-père »). En clair, ramener les tarifs d'électricité au niveau du Nord-Est américain signifie 1) la disparition des grandes industries «électrivores » au Québec d'ici la fin de l'amortissement de leurs actifs actuels et 2) la disparition du tiers de la contribution du secteur industriel au PIB québécois, part en effet que génèrent les industries "électrivores ». Les sceptiques demanderont : que perd-on vraiment comme société ? Après tout, nous dit-on, les redevances accrues à l'exportation d'électricité vont permettre de réduire la dette et - je cite - les « fonds libérés [...] serviront directement ou indirectement à recréer l'emploi ailleurs dans l'économie » (extrait du mémoire de Pierre Fortin auprès de la Commission sur l'énergie, 2005).

"Ailleurs dans l'économie », peut-être, mais ailleurs en région ? Cette création d'emploi se fera t'elle vraiment en région une fois qu'on y aura fermé les grandes entreprises énergivores? Ou alors, devons-nous comprendre qu'une fois fermés les établissements des grands employeurs privés en région, les «fonds libérés » permettront de financer la recherche et l'attraction de nouveaux investissements industriels en région? Ce serait un comble.

À la base de ces espoirs peu réalistes, il y a une méconnaissance des défis à la localisation d'entreprise que représente l’investissement en région éloignée. Il est en effet curieux de constater comme la question de l'impact de la hausse des tarifs d'électricité sur les régions n'est pas souvent étudiée au Québec. On balaie souvent la question en s'appuyant sur la faible part relative de l'emploi des entreprises «électrivores ». Il est pourtant pertinent de rappeler que plusieurs régions au Québec ont été à toutes fins pratiques créées par les grandes entreprises, et que si un tissu économique s’y est développé, c’est le plus souvent une activité induite par l'activité de ceux qui demeurent les principaux employeurs privés dans ces régions. Il y a en effet encore beaucoup de raisons de croire que les grandes entreprises continuent d'être aujourd'hui la justification économique de plusieurs villes et villages en région. Si les grandes entreprises ne sont plus présentes, combien de temps pourra-t-on justifier l'entretien des grandes infrastructures qu'elles utilisent? Les chemins de fer disparaîtront les premiers, les infrastructures portuaires suivront tôt ou tard. On l'a vu avec des infrastructures portuaires sur le fleuve Saint-Laurent. Au-delà d'un certain état de non-entretien, il y a une dégradation puis une rupture définitive.

Avec une hausse marquée des tarifs d'électricité, avec la fermeture graduelle des industries "électrivores ", les économies régionales entreront dans une spirale 
d'appauvrissement. On peut rêver à une intervention massive de l'État central. Mais est-il réaliste de croire que les contribuables des grands centres, qui jouissent d'économies plus robustes parce que plus diversifiées, voudront subventionner sans fin le niveau de vie des populations en région?

\section{Que peut-on faire?}

Ce risque d'appauvrissement, comment peut-on faire pour l'éliminer, pour le réduire ?

Il y a quelques voies de solution bien connues telles que la transformation en aval et la diversification économique.

La transformation accrue sur place ? Les possibilités varient selon le secteur. Depuis les difficultés d'exportation de bois d'œuvre aux États-Unis, nous constatons au Québec une multiplication des projets de deuxième et de troisième transformation du bois (ex. : meubles et matériaux de construction spécialisés) non sujets à des blocages sur nos marchés d'exportation. Nous disposons de ressources et de savoir-faire et, manifestement, d'un grand nombre d'entrepreneurs capables de relever le défi. La situation est beaucoup plus préoccupante pour les produits métalliques. La réalité est que la disponibilité de métal primaire n'est pas un facteur de localisation dans la grande majorité des cas et que la capacité d'entrepreneurs de démarrer de telles activités en région a été jusqu'ici très réduite. C'est un secteur où nous avons peu d'expérience et de tradition et où, pas plus qu'ailleurs, on ne peut s'improviser.

La diversification économique en dehors de l'industrie principale de la région est-elle une solution possible ? Oui, mais attention. Il ne s'agit pas uniquement de diversification radicale, bien que souhaitable lorsqu'elle est possible. La voie la plus réaliste m’apparaît la diversification que je qualifierais de "liée ", c’est-à-dire liée aux activités industrielles en place. Cette diversification table sur les savoirs générés dans l'activité principale, les savoirs qui peuvent se décliner de nouvelles façons avec de nouvelles technologies. Je n'en donnerai que deux exemples.

- Il peut s’agir de nouvelles méthodes de planification environnementale et d'optimisation de la ressource primaire au début du processus; je pense aux travaux qui se font au Québec en ligniculture, impliquant le développement d'essences à croissance rapide. Il y a actuellement, et c'est récent, plus de 40 projets de recherche sur ce sujet au Québec dans six universités et aussi dans les plus grandes entreprises et impliquant des approches radicalement nouvelles telles que la génomique forestière.

- Il peut s'agir de logiciels de simulation et de contrôle, tant au point de vue des concepts que des outils informatiques (je pense entre autres aux nouvelles possibilités qu'offrent les applications de l'informatique en temps réel pour les contrôles de processus industriels de grande envergure telles que la métallurgie primaire, les pâtes et papiers et, pourquoi pas, la tarification électrique en temps réel).

\section{La voie la plus réaliste m’apparaît la diversification que je qualifierais de « liée », c'est-à-dire liée aux activités industrielles en place.}

L'émergence récente d'une industrie d'équipementiers québécois reliés à l'aluminium illustre ce phénomène de diversification liée.

Si l'on arrive à mieux marier ces nouvelles technologies à notre patrimoine industriel, c'est le Québec qui sera gagnant, celui des régions comme celui des grands centres. Cette base de connaissance, existant déjà dans les usines, est le point de départ. Il y a de bonne chance qu'elle soit transférable. Comment peut-on l'identifier ? La formaliser ? La commercialiser ? Et puis, quant aux entrepreneurs : comment les dépister, les financer, les accompagner ? Ce sera réalisable dans la mesure où il y aura un véritable système d'innovation ouvert sur l'extérieur. Un système d'innovation où, au-delà des infrastructures pourtant nécessaires (centres de R\&D, universités), il y aura un esprit de coopération, une fluidité et j'irais jusqu'à dire, une générosité dans les échanges.

Et pourtant, rien n'est possible sans un meilleur voisinage entre les communautés et les grandes entreprises. La qualité de ce voisinage implique trois principes :

- d'abord, la responsabilité, sans quoi la confiance se trouve minée, et les communications limitées à 
des négociations serrées et interminables plutôt qu’à de véritables échanges créatifs;

- ensuite le respect; la société civile et les entreprises ont toutes deux des raisons d'agir comme elles le font; le respect entraîne l'effort de connaître l'autre partie, et d'être conscient de leurs contraintes;

- mais surtout : la relation; la relation implique une ouverture dans les communications, une communication quant aux attentes respectives, une relation qui amène un engagement réciproque; une relation avec ce que cela suppose d'effort de stabilité.

Ces trois principes peuvent paraître d'une banalité désolante. À la marge, ils peuvent pourtant faire la différence entre une région propice à l'innovation et à l'investissement et une région qui ne l'est pas; toutes les régions ne présentent pas le même profil à cet égard. Comme je l'ai mentionné, ce sont les facteurs intangibles qui peuvent être déterminants.

\section{Je me permets aussi de questionner la pérennité de la manne attendue quant aux tarifs à l'exportation.}

\section{Conclusion}

J'ai donc évoqué la crise de l'énergie dans laquelle nous nous trouvons, les contraintes auxquelles les entreprises doivent faire face, les attentes légitimes des communautés d'accueil. Je résume à présent en cinq points les éléments à considérer dans l'avenir :

- L’énergie - sa disponibilité et des prix compétitifs

- constituera un facteur de localisation encore plus important que par le passé pour au moins les 15 à 20 prochaines années. Est-ce vraiment le bon moment pour le Québec et ses régions d'éliminer cet avantage étant donné le potentiel énergétique qui demeure au Québec ?

- La relation entre la communauté d'accueil et l'entreprise "électrivore ». L’énergie sera donc un facteur de localisation critique, mais pas à n'importe quelle condition. La capacité de créer et de maintenir une relation entre l'entreprise et la communauté d'accueil est absolument essentielle.
Sans relation saine, sans le respect et la responsabilité qui l'accompagnent, les chances de succès sont sérieusement compromises.

- Le long terme. Celui-ci découle du point précédent, mais il vaut la peine d'être distingué dans le contexte actuel. Sans prise en compte des intérêts à long terme des parties concernées, l'échec est prévisible. Ce serait d'autant plus dommage de sacrifier le patrimoine industriel du Québec à cause d'une crise conjoncturelle dans nos disponibilités énergétiques. À cet égard, je me permets aussi de questionner la pérennité de la manne attendue quant aux tarifs à l'exportation. Avec une cinquantaine de nouvelles centrales énergétiques prévues aux États-Unis chaque année au cours des dix à quinze prochaines années, la prime actuelle à l'exportation va t'elle demeurer ? Il serait malheureux de sacrifier un siècle de développement industriel en région pour ce qui est en fait une spéculation sur la durée de cette prime.

- La diversification liée. La «diversification liée » est une piste de création de richesse plus probable que la diversification radicale. L'identification des occasions doit partir des besoins mal comblés, mais elle peut aussi provenir d'applications transversales reliées à d'autres disciplines. Cette diversification est difficile à planifier d'office, mais plusieurs occasions d'affaires créées dans votre région depuis quelques années suggèrent que cela est possible. Comment accélérer ce mouvement ? Ceci me mène au point suivant.

- La dynamique d'innovation régionale. Nous avons fait une étude en 2005 auprès des grands experts internationaux du soutien à l'innovation, du Japon jusqu'à la Suède, et la conclusion est claire. Pour que les régions se développent, pour qu'elles innovent puisque le développement dans un monde concurrentiel passe par l'innovation, cela suppose, au-delà d'infrastructures et de spécialistes, au-delà d'une ouverture intense sur l'extérieur, des liens forts et durables entre les équipes scientifiques, commerciales et politiques de la région. Cela prend un leadership fort, non pas un leadership d'autorité mais un leadership de facilitation et de coordination et qui s'exerce dans la durée. Est-ce qu'une telle dynamique existe chez vous ? Est-ce qu'elle peut être développée ? 
Je vous donnerai comme exemple une initiative employée en Suède pour encourager la diversification économique des régions par le biais du financement de leur système d'innovation. Ce programme national est particulier à plus d'un titre :

- la durée sur laquelle elles sont accordées (dix ans), évitant donc le piège des actions à court terme (pour ne pas dire à courte vue), évitant le piège de la dispersion et du « saupoudrage »;

- l'attribution aux regroupements d'intervenants régionaux (entreprises, gouvernements locaux, institutions de formation) qui peuvent monter un projet bien structuré;

- la nature concurrentielle du programme. Les fonds sont en effet accordés aux régions présentant la cohésion la plus forte.

Si ce programme était en vigueur au Québec, et que les régions étaient en compétition entre elles, votre région l'emporterait-elle?

Vous me direz peut-être que tout ce débat est un débat d'arrière-garde et que notre avenir économique sera fait de technologies d'information et de santé. À cet égard, il est utile de noter que quelque chose d'étonnant se produit au Canada. Dans les régions éloignées des grands centres, les projets industriels se multiplient, des projets qui ne relèvent pas du pharmaceutique, des télécommunications ou de l'aéronautique, de la haute technologie, de la soi-disant «nouvelle économie ». Mais qui concernent des produits comme le cuivre et le magnésium, le diamant, l'uranium, le pétrole, des métaux rares ou précieux comme l'or et le platine, le niobium, le molybdène ou le vanadium. Des projets de grande envergure comme l'ouverture de nouvelles mines, la construction de pipelines et les terminaux méthaniers, et les développements industriels qu'ils rendent possibles. En fait, les trois quarts de la valeur des projets d'investissement industriel au Canada concernent les ressources ou leur première transformation et ont lieu non pas à Montréal ou à Toronto, mais dans des régions comme la vôtre, à six, dix ou douze heures des grands centres urbains. Il s'agit dans les faits d'une deuxième vague d'industrialisation des régions au Canada. Celle-ci se fera différemment de la précédente, mais ce qui est sûr, c'est que l'énergie continuera de jouer un rôle majeur. Voudrez-vous faire partie de cette nouvelle industrialisation? À quelles conditions?

\section{Note}

1 Jean Matuszewski est président de E\&B DATA (www. ebdata.com), chef de file en matière de données économiques et d'affaires. Avec ses 25 professionnels, E\&B DATA est dans certains domaines d'information, dont les secteurs de pointe et les investissements industriels, la meilleure référence canadienne. Ses interventions permettent le renouvellement des interventions en matière de développement industriel et régional. 


\section{Publicité}

\section{Maîtrise en gestion de projet}

